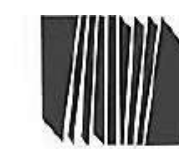

UNIVLRSIDADPEUAGOGICA EXPLR HALNTAL LIBCR TROO

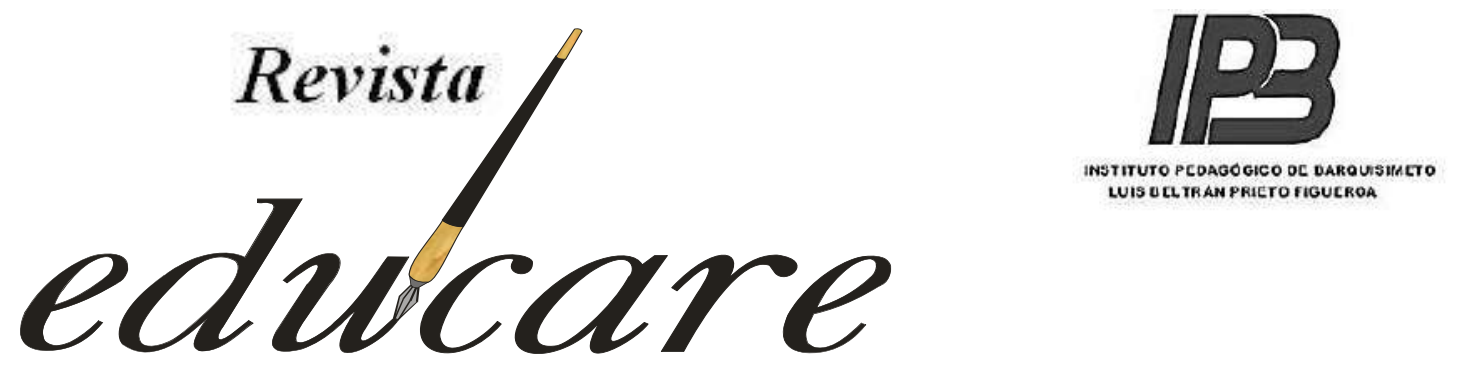

Órgano Divulgativo de la Subdirección de Investigación y Postgrado del Instituto Pedagógico de Barquisimeto "Luis Beltrán Prieto

Figueroa"

RARQUISUUEIO - EDO. LARA - VEMEZUELA

\title{
INTELIGENCIA EMOCIONAL EN ESTUDIANTES VENEZOLANOS DE EDUCACIÓN MEDIAY Y UNIVERSITARIA
}

\author{
EMOTIONAL INTELLIGENCE IN VENEZUELAN STUDENTS \\ IN HIGH SCHOOL AND COLLEGE
}

\section{DEPOSITO LEGAL: ppi201002LA3674 ISSN: 2244-7296}

\author{
Jesús Marcos Segura Martín *
}

Universidad Nacional de Educación a Distancia (UNED-España). 


\begin{tabular}{|c|c|}
\hline TRABAJO DE INVESTIGACIÒN & Jesús Marcos Segura Martín * \\
\hline Recibido:22-01-2016 & Aceptado: 12-07-2016 \\
\hline RESUMEN & ABSTRACT \\
\hline $\begin{array}{l}\text { El conocimiento de las habilidades } \\
\text { emocionales de nuestros estudiantes y los factores } \\
\text { que influyen sobre las mismas, permitirá mejorar } \\
\text { el proceso de enseñanza y aprendizaje. La } \\
\text { presente investigación tiene por objetivo } \\
\text { diagnosticar las habilidades emocionales de } \\
\text { estudiantes venezolanos de educación media y } \\
\text { universitaria para determinar relaciones entre } \\
\text { habilidades emocionales y los efectos del género, } \\
\text { contexto geográfico y edad en dichas habilidades } \\
\text { emocionales. Hemos realizado una investigación } \\
\text { con diseño de campo. Los niveles de } \\
\text { investigación fueron exploratorio, descriptivo y } \\
\text { correlacional. La población estuvo conformada } \\
\text { por estudiantes de educación media y } \\
\text { universitaria. La muestra fue intencional de } 441 \\
\text { alumnos. El instrumento de recogidas de datos } \\
\text { fue un cuestionario de emociones. Los principales } \\
\text { hallazgos de la investigación revelan la existencia } \\
\text { de diferencias estadísticas significativas entre las } \\
\text { habilidades emocionales de los estudiantes por } \\
\text { género, existe relación estadísticamente } \\
\text { significativa entre las habilidades emocionales y } \\
\text { las habilidades emocionales de autocontrol y } \\
\text { competencia social fueron las menos favorecidas. } \\
\text { Palabras clave: Inteligencia emocional; } \\
\text { habilidades emocionales; estudiantes de } \\
\text { educación media. }\end{array}$ & $\begin{array}{l}\text { Knowledge of our students' emotional } \\
\text { abilities and the factors that influence them will } \\
\text { improve the teaching and learning process. The } \\
\text { present research aims to diagnose the emotional } \\
\text { abilities of Venezuelan High School and } \\
\text { university students to determine relationships } \\
\text { between emotional abilities and the effects of } \\
\text { gender, geographic context and age in these } \\
\text { emotional abilities. We have done research with } \\
\text { field design. The levels of research were } \\
\text { exploratory, descriptive and correlational. The } \\
\text { population was made up of students of High } \\
\text { School education and university. The sample } \\
\text { was constituted by } 441 \text { students. A } \\
\text { questionnaire of emotions was the instrument of } \\
\text { data collection. The main findings of the } \\
\text { research reveal the existence of significant } \\
\text { statistical differences between students' } \\
\text { emotional abilities by gender, there is a } \\
\text { statistically significant relationship between } \\
\text { emotional abilities and emotional skills of self- } \\
\text { control and social competence were the least } \\
\text { favored. }\end{array}$ \\
\hline
\end{tabular}

${ }^{*}$ Centro de Recuperación Académica para Ciencias (CRAC-Venezuela). jmsegur@yahoo.es. Universidad Nacional de Educación a Distancia (UNED-España). 


\section{INTRODUCCIÓN}

El Informe sobre Desarrollo Humano 2013 del Programa de las Naciones Unidas para el Desarrollo (PNUD), señala que la tasa de homicidios en la República Bolivariana de Venezuela es de 45,1 por cada 100 mil habitantes (el valor promedio en América Latina y del Caribe es 22,2). Según el diario El Universal de Venezuela (2013, Mayo 30), los índices de violencia que azotan actualmente a Venezuela han venido afectando directamente a los jóvenes entre 0 y 17 años. En las entidades donde hay mayor población, existe mayor incidencia delictiva en los niños, niñas y adolescentes, que en muchos de los casos terminan en muertes violentas. El mismo diario señaló que los homicidios son la primera causa de muerte en jóvenes venezolanos. El estado Miranda es el segundo estado después del Distrito Metropolitano de Caracas con los índices más altos de crímenes violentos y homicidios en Venezuela.

En nuestra experiencia docente en las instituciones que participaron en esta investigación y en otras instituciones pertenecientes a Los Teques y al Distrito Metropolitano de Caracas, hemos observado una serie de dificultades como, por ejemplo, bajo rendimiento académico, clases bulliciosas, alumnos poco disciplinados, elevado número de inasistencias, violencia verbal y no verbal entre alumnos, peleas por cosas insignificantes, llanto y nerviosismo a la hora de presentar exámenes o presentaciones orales, embarazo precoz... En nuestra opinión, algunas de estas conductas sugieren la carencia de una o más habilidades emocionales. Con referencia a lo anterior, valores bajos del autocontrol y competencia social, entre otras, son asociadas con las dificultades citadas (Gilar, Miñano y Castejón, 2008). Creemos que dentro de las estrategias docentes para la escuela del siglo XXI, es necesario incluir el desarrollo de las habilidades emocionales de los discentes, profesores y directivos para otorgarles las herramientas que facilitan la comunicación y el desarrollo del proceso enseñanza y aprendizaje del alumnado. Las emociones son uno de los factores más importantes de la conducta del ser humano y, en un gran número de ocasiones, lo que hacemos está determinado más por nuestras emociones que por la razón (Goleman, 1995). La competencia emocional es factor esencial para la prevención, el desarrollo personal y social y una competencia básica para la vida (Alonso y Gallego,1999; Bisquerra, 2003). En el orden de las ideas anteriores, Caplanetal. (1992) y 
Cohen (1999), citados por Vandervoort (2006), señalan que la inclusión de clases de inteligencia emocional en el plan de estudios de la escuela primaria y secundaria ha sido eficaz en el aumento de la inteligencia emocional, la reducción de los problemas emocionales y del comportamiento que pueden interferir con el proceso de aprendizaje. El desarrollo intelectual, emocional y el rendimiento académico son interdependientes. Estudiantes con elevada inteligencia emocional tienden a ser más prosocial, tienen un mejor rendimiento escolar y mejor comportamiento. Las sensaciones y las emociones positivas pueden aumentar grandemente el proceso de aprendizaje; pueden mantener al principiante en la tarea y pueden proporcionar un estímulo para el nuevo aprendizaje. Asimismo, conductas como el abandono escolar, emociones negativas, el bajo rendimiento, consumo de drogas y la delincuencia juvenil se han relacionado con la ausencia de competencias sociales (Serrano, 2006; Gil-Olarte et al., 2006; Kimbrough, 2008; Ruiz, 2008).

El desarrollo de la inteligencia emocional en nuestros centros educativos es de vital importancia, debido a que contribuye a mejorar las bases para una mejor convivencia escolar. La inteligencia emocional también es importante para una comunicación gerencial efectiva dentro de las instituciones educativas. Así, por ejemplo, Singh y Manser (2008) investigaron la relación entre la inteligencia emocional de los directores de instituciones educativas y la satisfacción de los educadores en su ambiente colegial. Los resultados de esta investigación indicaron que los educadores se sentirían satisfechos en la escuela si sus directores crearan la oportunidad de desarrollar sus capacidades en un ambiente que consolide la comunicación eficaz, relaciones sanas, empatía, confianza y desean ser dirigidos por los directores que se sienten confiados en su papel de director, que mantienen autodominio, son adaptables, flexibles y que hace frente al futuro con optimismo. En este sentido, profesores y directivos deben tener empatía, autocontrol, autoconciencia, motivación y habilidades sociales interpersonales. Estas habilidades emocionales son fundamentales en el profesor para que pueda desarrollar la inteligencia emocional de sus alumnos. Una buena gestión de las habilidades emocionales puede mejorar la motivación y la energía de un profesor, y despertar el interés y atención de los alumnos

Existen pocas dudas de que para ser exitoso se deben desarrollar las habilidades de comunicación efectiva, gestionar la interacción social y las relaciones sociales. El 
desarrollo de las habilidades emocionales es un medio idóneo para aumentar la capacidad de asimilación y comprensión de las interacciones humanas y dar sentido a las acciones y relaciones con otras personas (Lozano et al., 2010). Cuanto más experto sea el profesor en gestionar sus emociones mejor entenderá las necesidades de los estudiantes, forma una fuerte unión con sus estudiantes y colegas, reduce la tensión, gestiona mejor el tiempo y alcanza sus metas (Neptune, 2008; Riggio y Reichard, 2008). En este mismo sentido, Fernández-Berrocal y Extremera (2009) señalan la necesidad de construir espacios educativos que hagan crecer las fortalezas individuales del alumnado para su crecimiento personal y social. Pero para ello la escuela no puede funcionar como un oasis emocional en un entorno cerrado al cambio, sino como un centro dinamizador que trascienda su contexto y se extienda a la familia y a la sociedad. En este propósito, debemos señalar que la capacidad para identificar, comprender y regular las emociones es fundamental entre el profesorado, ya que estas habilidades van a influir en los procesos de aprendizaje, en la salud física, en la calidad de las relaciones interpersonales, en el rendimiento académico y laboral. Profesores emocionalmente inteligentes tienen en sus manos la capacidad de tratar con mayor éxito los contratiempos cotidianos a los que se enfrenta en el aula y promueve en otros sensaciones de seguridad para la resolución de problemas (Pérez, 2005; Castejón et al., 2008 y Cabello et al., 2010).

Para Soriano y Franco (2010), una adecuada competencia emocional está asociada con niveles más altos de felicidad actual, mayor porcentaje de felicidad previa, niveles más elevados de afecto positivo, mayor satisfacción vital, mayor autoestima. Además, una adecuada competencia emocional presenta una relación negativa con los niveles de depresión y ansiedad.Diversas investigaciones resaltan la importancia de desarrollar la educación emocional de docentes y discentes (Palomera, Fernández-Berrocal y Brackett, 2008; Pena y Repetto, 2008; Soriano y Osorio, 2008; Molero, Ortega y Moreno, 2010). Las habilidades emocionales y sociales están relacionadas con el éxito y pueden ser mejoradas a través de programas de entrenamiento.Sin embargo, debido a que en nuestro sistema educativo ha existido un mayor interés por los aspectos cognitivos del aprendizaje que por el desarrollo de las habilidades emocionales de los discentes, encontramos pocos programas escolares y universitarios para educar las habilidades emocionales y desarrollar la inteligencia emocional de nuestros estudiantes. Según Gutiérrez (2010), la generalidad 
del alumnado de magisterio reconoce que la temática de las habilidades emocionales les ayuda enormemente en su formación como maestros, al tiempo que les ayuda a crecer personalmente. Siempre resulta ser un tema novedoso para los alumnos y nunca contemplado en los currículos de las diferentes etapas educativas por las que han pasado. Cabe agregar que faltan investigaciones que relacionen la inteligencia emocional de Goleman y las teorías de los estilos de aprendizaje. También faltan investigaciones que relacionen las inteligencias múltiples de Gardner (2001) y los estilos de aprendizaje (Alonso y Gallego, 2004).

Elizondo (2011), al referirse a las competencias que debe tener un director académico universitario, considera que las habilidades emocionales de autoconciencia y empatía, son intrínsecos a la manera como un director académico manifiesta los talentos de relaciones humanas y ayudan al director a desarrollarse en una persona más psicológicamente saludable y mejor en términos generales, permitiendo el desarrollo de los objetivos educativos en un clima apropiado para el desarrollo integral del estudiantado.

Sobre la base de las consideraciones anteriores, se deduce que el conocimiento de la inteligencia emocional de nuestros estudiantes permitirá predecir las habilidades emocionales de los estudiantes y, por tanto, formular estrategias que aumenten los logros académicos de los estudiantes, mejorar el comportamiento y la calidad de las relaciones que rodean a cada estudiante en su entorno educativo. El desarrollo de habilidades emocionales en entornos educativos permitirá mejorar la convivencia escolar y no escolar entre la población joven y su rendimiento académico. En efecto, las investigaciones realizadas en la última década indican que la inteligencia emocional puede ser la influencia más importante para tener éxito en el trabajo y en nuestra vida personal, y sostienen que la interacción de los dominios cognoscitivos y emocionales desarrolla un aprendizaje eficaz.

En la literatura podemos encontrar diversas definiciones de inteligencia emocional (Salovey y Mayer, 1990; Goleman, 1998; Alcázar, 2001; Caruso y Solovey, 2005; Fernández-Berrocal, Extremera y Palomera, 2008; Segura, 2011). Todas estas definiciones no son excluyentes sino que se complementan unas a otras. En esta investigación nos basamos sobre el modelo de Goleman (1998) para definir la inteligencia emocional como un tipo de inteligencia que muestra la posesión de habilidades de autoconciencia, empatía, 
motivación, autocontrol y competencia social, indispensables para resolver y precaver problemas y, por tanto, alcanzar un mayor desarrollo personal y profesional.

Cabe decir que se observa en la literatura sobre inteligencia emocional un gran interés en la elaboración de modelos e instrumentos de evaluación de la inteligencia emocional. En efecto, existen diversos cuestionarios diseñados con el objeto de medir la inteligencia emocional. Trujillo y Rivas (2005), Pérez, Petrides y Furnham (2007) hicieron una revisión de diversos cuestionarios para medir la inteligencia emocional y modelos de inteligencia emocional. En esta investigación, el instrumento de recogida de datos ha sido un cuestionario de emociones basado sobre el modelo de Goleman (1998), adaptado al vocabulario de nuestros estudiantes y validado siguiendo diferentes estrategias para determinar, comparar y analizar las habilidades emocionales de 432 estudiantes venezolanos de bachillerato y formación técnica superior, provenientes de cuatro instituciones de Caracas y dos instituciones de Los Teques (Estado Miranda).

\section{MÉTODO}

\section{Objetivos}

Diagnosticar las habilidades emocionales de estudiantes venezolanos de educación media y universitaria para determinar relaciones entre habilidades emocionales y los efectos del género, contexto geográfico y edad en dichas habilidades emocionales.

\section{Hipótesis de la investigación}

Hipótesis a. Existen diferencias significativas en las habilidades emocionales de los estudiantes.

Hipótesis b. Existe correlación entre las habilidades emocionales de los estudiantes.

Hipótesis c. Existen diferencias significativas entre las habilidades emocionales de los estudiantes por contexto geográfico.

Hipótesis d. Existen diferencias significativas entre las habilidades emocionales de los estudiantes por género.

Hipótesis e. Los estudiantes de género femenino son emocionalmente más inteligentes que los de género masculino. 
Hipótesis f. Existe correlación lineal significativa entre las habilidades emocionales de los estudiantes y sus edades.

\section{Tipo de investigación}

Los criterios empleados en este estudio para establecer la metodología de investigación utilizada fueron: diseño de investigación y nivel de investigación.Se realizó una investigación con diseño de campo-cuantitativo, apoyada en cuestionarios.

La investigación es cuantitativa debido a que se utilizó un procedimiento formal, objetivo y sistemático para obtener datos numéricos correctos y adecuados, que luego fueron sometidos a los análisis estadísticos correspondientes aplicándose técnicas estadísticas inferenciales (Burns y Grove, 2005 y Salazar, 2002).

La investigación fue de campo, debido a que la recolección de datos se realizó directamente de la realidad donde ocurrieron los hechos, sin manipular o controlar variable alguna (Universidad Pedagógica Experimental Libertador [UPEL], 2006).

La investigación posee un nivel descriptivo, debido a que se midió una serie de características de un grupo de estudiantes y profesores en un momento y contexto determinado (Sampieri, Collado y Lucio, 2009). También es correlacional, debido a que se midió el grado de relación entre una o más variables.

\section{Población y muestra}

La población estuvo conformada por estudiantes de $3^{\mathrm{a}}, 4^{\mathrm{o}}$ y $5^{\mathrm{o}}$ año de educación media y estudiantes de los primeros semestres de formación universitaria de las especialidades de fisioterapia e informática pertenecientes a instituciones de Los Teques y Caracas.

Se utilizó una muestreo intencional o de conveniencia de 441 alumnos de educación media y formación técnica superior de edades entre 15-25 años. 9 cuestionarios de emociones fueron contestados de manera incompleta y fueron anulados. El total de cuestionarios de emociones analizados fue 432 (193 mujeres y 239 varones). 62 alumnos fueron de los primeros semestres de formación técnica superior. +199 cuestionarios fueron aplicados en los Institutos Fernando de Magallanes, José Vicente de Unda y San Martín de Porres. 233 cuestionarios fueron aplicados en las otras instituciones, previa autorización y colaboración de la dirección de las respectivas instituciones. A los profesores y alumnos que tomaron el cuestionario, se les explicaron los objetivos del cuestionario y la forma de 
contestarlo. Para realizar esta investigación se seleccionaron el Instituto Victegui de Los Teques (secciones $4^{\circ} \mathrm{A}, 4^{\circ} \mathrm{B}, 5^{\circ} \mathrm{A}$ y $5^{\circ} \mathrm{B}$ de educación media), el Colegio Universitario de Los Teques Cecilio Acosta (CULTCA) (alumnos de las secciones $4^{\circ}$ y $5^{\circ}$ de $\operatorname{los}$ primeros semestres de la carrera de fisioterapia), el Instituto de Ciencias Náuticas Fernando de Magallanes de Caracas (secciones $4^{\circ} \mathrm{H}, 4^{\circ} \mathrm{I}, 5^{\circ} \mathrm{J}$ y $5^{\circ} \mathrm{K}$ de educación media), el Liceo José Vicente de Unda de Caracas (secciones $3^{\circ}, 4^{\circ}$ y $5^{\circ}$ de educación media), al Colegio San Martín de Porres de Caracas (secciones $3^{\circ}, 4^{\circ}$ y $5^{\circ}$ de educación media) y el Instituto Universitario de Tecnología Industrial Rodolfo Loero Arismendi con sede en Los Chaguaramos de Caracas (alumnos de los dos primeros semestres de la carrera de informática). Salvo CULTCA todas las otras instituciones son privadas. La selección de estas instituciones fue basada sobre su representatividad en las zonas educativas a las que pertenecen y al ser profesor en algunas de estas instituciones nos facilitaba la aplicación del cuestionario de emociones y realizar un seguimiento a los alumnos.

Los resultados obtenidos fueron contrastados con las opiniones emitidas por el personal de aula y administrativa de amplia experiencia docente. El número de entrevistas realizadas fue 20. La pregunta realizada fue ¿Piensa $U d$. que nuestros alumnos presentan dificultades en las habilidades emocionales de autocontrol y competencia social?

\section{Instrumento de recogida de datos}

El instrumento de recogida de datos fue un cuestionario de emociones basado sobre el modelo de Goleman (1998) y validado siguiendo diferentes estrategias. Para evaluar las habilidades emocionales de empatía, motivación, competencia social, autoconciencia del primer nivel, autoconciencia del segundo nivel y autocontrol, el cuestionario fue organizado en cinco ítems por habilidad emocional para un total de 30 ítems, los cuales se responden sobre una escala del tipo Likert de 5 puntos. Todos los ítems están distribuidos aleatoriamente. Los resultados del cuestionario se obtienen sumando los puntos correspondientes a cada ítem por habilidad emocional, siendo el máximo de puntos por habilidad emocional 25. La puntuación de cada ítem por habilidad emocional se plasma en una hoja que sirve para determinar la puntuación total de cada habilidad emocional. Una muestra parcial de este cuestionario de emociones es mostrada en la tabla 1. En Segura (2011:94-96), se puede encontrar información más detallada sobre el mismo. 
Tabla 1. Cuestionario de habilidades emocionales

Lea cuidadosamente cada una de las siguientes oraciones y realice su autoevaluación de acuerdo con la siguiente escala. Sea lo más sincero posible en su autoevaluación.

Si está totalmente en desacuerdo marque 1 . Si está en desacuerdo marque 2.

Si no está de acuerdo ni en desacuerdo marque 3 . Si está de acuerdo marque 4. Si está totalmente de acuerdo marque 5.

1. Soy consciente de las reacciones físicas (gestos, dolores, cambios súbitos) que señalan una reacción impulsiva o visceral.

2. Admito de buena gana mis errores y me disculpo.

3. No me aferro a los problemas, enfados o heridas del pasado, y soy capaz de dejarlos atrás para avanzar.

4. Normalmente, tengo una idea exacta de cómo me percibe la otra persona durante una interacción específica.

5. Hay varias cosas importantes en mi vida que me entusiasman, y lo hago patente.

6. Tengo facilidad para conocer e iniciar conversaciones con personas desconocidas cuando tengo que hacerlo.

7. Me tomo un descanso o utilizo otro método activo para incrementar mi energía cuando noto que mi nivel de energía está decayendo.

8. No me cuesta demasiado asumir riesgos, pero con cierto grado de prudencia, es decir, asumir riesgos prudentes.

9. Me abro a las personas en la medida adecuada, no demasiado, pero lo suficiente como para no dar la impresión de ser frío y distante.

10. Puedo participar en una interacción con otra persona y captar bastante bien cuál es su estado de ánimo sobre la base a las señales no verbales que me envía.

- Escriba sus puntuaciones para cada pregunta numerada en la categoría correspondiente.

- Sume las puntuaciones de cada categoría para obtener el total de ese factor específico de CE

\begin{tabular}{|l|l|}
\hline Autoconciencia: & Empatía \\
\hline 1 & 4 \\
\hline 7 & 10 \\
\hline 13 & 16 \\
\hline 19 & 22 \\
\hline 25 & 28 \\
\hline Total autoconciencia: & Total de empatía: \\
\hline
\end{tabular}

Los datos fueron analizados e interpretados mediante el programa estadístico SPSS y

Past 3. Se hicieron ficheros para cada sección investigada en cada institución y 
posteriormente fueron convenientemente fusionados para el análisis total. En todas las situaciones, se realizó un estudio exploratorio de normalidad aplicando las siguientes pruebas de normalidad: Test de Kolmogorov-Smirnov, histogramas, gráficos Q-Q de normalidad, diagramas de cajas y bigotes, Shapiro-Wilk, Anderson-Darling y Jaque-Bera. Los resultados de estas pruebas de normalidad indicaron que los datos no seguían una distribución normal y fueron tratados mediante pruebas no paramétricas para comparar medianas y determinar correlaciones entre variables. En los contrastes de hipótesis realizados, un valor de $p<0,05$ fue tomado como una indicación de relación significativa entre variables o diferencia significativa entre las medianas de las variables. Un valor de $p>$ 0,05 fue tomado como una indicación de igualdad de medianas o relación no significativa entre las variables. El cuestionario fue sometido a pruebas de validez de datos, fiabilidad, análisis factorial y la opinión de expertos de la UNED de España.

\section{Validación del cuestionario de emociones}

Todos los datos, casos o variables pasaron las comprobaciones de validez. El análisis de fiabilidad de las seis habilidades emocionales o los seis elementos que constituyen el cuestionario a partir de las medias totales de sus treinta ítems, obtenidos con 432 cuestionarios, indicó un valor de fiabilidad alfa de Cronbach de 0,859. Este valor es bueno para garantizar la fiabilidad del cuestionario

El cuestionario posee los siguientes parámetros favorables para un Análisis Factorial. La matriz de correlación posee valores de significancia unilateral de 0,000, valor del determinante bajo (0,090), prueba de esfericidad de Bartlett con un nivel crítico de significancia de 0,000, medida de adecuación muestral de Kaiser-Meyer-Olkin de 0,886 (se considera que un valor superior o igual a 0,8 es meritorio) y matrices anti-imagen favorables (en todos los casos se obtuvieron valores superiores a 0,86 en la diagonal de la matriz de correlación anti-imagen).

Para explicar el 79,08\% de la varianza total fue requerido extraer tres factores. El $36,337 \%$ corresponde al primer factor, $21,954 \%$ al segundo factor y $20,728 \%$ al tercer factor. Estudiando las comunalidades obtenidas por el método de extracción (análisis de componentes principales), pudimos valorar que la variable empatíafue la peor explicada y: el modelo solo es capaz de reproducir el $63,3 \%$ de su variabilidad original. Los otros 
valores obtenidos fueron: Autoconciencia 1: 0,968; Autoconciencia 2: 0,708; Autocontrol: 0,945; Motivación: 0,660 y Competencia social: 0,828.

La matriz de componentes rotados (método Varimax) permitió correlacionar el primer factor con competencia social $(0,893)$, autoconciencia $2(0,65)$, motivación $(0,652)$ y empatía $(0,636)$. El factor 2 está correlacionado con autocontrol $(0,942)$ y el tercer factor está correlacionado con autoconciencia $1(0,914)$. Como podemos observar se obtuvieron en todos los casos factoriales superiores al 63\%. Estos resultados son consistentes con los obtenidos en una investigación anterior, realizada con una muestra de 294 estudiantes (Segura, 2011: 99).

\section{RESULTADOS Y DISCUSIÓN}

\section{Prueba de normalidad}

En la tabla 2, se muestran los resultados para algunas de las pruebas de normalidad realizas. En la tabla 3, se muestran los promedios y medianas de las habilidades emocionales, las desviaciones típicas, asimetría y curtosis.

Para realizar el contraste de hipótesis estadística hemos planteado las siguientes hipótesis nula y alternativa.

Hipótesis nula $\mathrm{H}_{\mathrm{o}}$ : Los datos siguen una distribución normal.

Hipótesis alternativa $\mathrm{H}_{\mathrm{a}}$ : Los datos no siguen una distribución normal.

Rechazamos la hipótesis nula cuando $p<0,05$.

Como se observa en la figura 1 y a través de las pruebas de normalidad, citadas en la sección 2.5, no se cumple la hipótesis nula, es decir, los datos no siguen una distribución normal y deben ser tratados siguiendo pruebas no paramétricas.

Tabla 2. Pruebas de normalidad

\begin{tabular}{|l|c|c|c|c|c|c|}
\hline \multirow{2}{*}{ Prueba } & $\begin{array}{c}\text { Autocon- } \\
\text { ciencia 1 }\end{array}$ & $\begin{array}{c}\text { Autocon- } \\
\text { ciencia 2 }\end{array}$ & $\begin{array}{c}\text { Autocon- } \\
\text { trol }\end{array}$ & Empatía & Motivación & $\begin{array}{c}\text { Competencia } \\
\text { Social }\end{array}$ \\
\hline \multirow{2}{*}{ Shapito-Wilk } & 0,9258 & 0,9358 & 0,9516 & 0,9353 & 0,9059 & 0,9605 \\
\cline { 2 - 7 } & $p=0,000$ & $p=0,000$ & $p=0,000$ & $p=0,000$ & $p=0000$ & $p=0,000$ \\
\hline \multirow{2}{*}{$\begin{array}{l}\text { Anderson- } \\
\text { Darling }\end{array}$} & 4,815 & 4,585 & 3,638 & 4,36 & 6,79 & 2,58 \\
\cline { 2 - 7 } & $p=0,000$ & $p=0,000$ & $p=0,000$ & $p=0,000$ & $p=0000$ & $p=0,000$ \\
\hline \multirow{2}{*}{ Jaque-Bera } & 432,8 & 287,5 & 149,9 & 276,5 & 594,6 & 112,17 \\
\cline { 2 - 7 } & $p=0,000$ & $p=0,000$ & $p=0,000$ & $p=0,000$ & $p=0000$ & $p=0,000$ \\
\hline
\end{tabular}


Tabla 3. Medianas, medias, asimetría y curtosis de las habilidades emocionales de estudiantes venezolanos

\begin{tabular}{|l|c|c|c|c|c|c|}
\hline $\begin{array}{l}\text { Habilidad } \\
\text { emocional }\end{array}$ & $\mathrm{N}$ & Mediana & Media & $\sigma$ & Asimetría & Curtosis \\
\hline Autoconciencia 1 & 432 & 19,5 & 19,21 & 3,47 & $-0,669$ & 0,820 \\
\hline Autoconciencia 2 & 432 & 19 & 18,74 & 3,28 & $-0,627$ & 0,634 \\
\hline Autocontrol & 432 & 18 & 18,12 & 3,46 & $-0,495$ & 0,151 \\
\hline Empatía & 432 & 19 & 18,77 & 3,38 & $-0,682$ & 0,860 \\
\hline Motivación & 432 & 20 & 20,13 & 3,20 & $-0,892$ & 1,432 \\
\hline Competencia Social & 432 & 18 & 18,14 & 3,47 & $-0,313$ & 0,063 \\
\hline
\end{tabular}

En relación a la hipótesis a. «Existen diferencias significativas en las habilidades emocionales de los estudiantes».

La hipótesis a fue contestada aplicando la prueba no paramétrica de Wilcoxon o prueba de dos muestras relacionadas para comparar las medianas dados en la tabla 3. Las muestras están relacionadas debido a que se aplicó el mismo cuestionario para determinar simultáneamente las habilidades emocionales de un mismo grupo de alumnos.

La prueba no paramétrica de Wilcoxon indicó que, salvo para las parejas empatíaautoconciencia $2(W=34404, z=0,59512, p=0,55176)$ y competencia social-autocontrol ( $W=39499, z=0,35099, p=0,7256$ ), existen diferencias significativas entre las otras habilidades emocionales. Por tanto, las habilidades emocionales de autocontrol y competencia social son las menos desarrolladas por los alumnos de educación media general y educación tecnológica, y no existen diferencias significativas entre las medianas de estas dos habilidades emocionales. La habilidad emocional con mayor puntuación fue motivación. En consecuencia, no se acepta la hipótesis de la investigación para las habilidades emocionales de empatía-autoconciencia 2 y competencia social-autocontrol, debido a que no se encontraron diferencias significativas. 
Un análisis por aula reveló que de las 20 aulas encuestadas, 17 aulas presentaron el menor promedio en la habilidad emocional de autocontrol. La segunda habilidad emocional con menor promedio fue competencia social.

En relación a la hipótesis b. «Existe correlación entre las habilidades emocionales de los estudiantes».

Para contestar la hipótesis b hemos utilizado el coeficiente de regresión no paramétrico de Rho de Spearman, dado en la tabla 4, y el siguiente contrate de hipótesis estadística:

Hipótesis nula: $\mathrm{H}_{\mathrm{o}}=$ No existe correlación entre las habilidades emocionales.

Hipótesis alternativa: $\mathrm{H}_{\mathrm{a}}=$ Existe correlación entre las habilidades emocionales.

Rechazamos la hipótesis nula si $p<0,05$.

Como podemos apreciar en la tabla 4, todos los valores de significancia son inferiores a $0,05 \mathrm{y}$, por tanto, se rechaza la hipótesis nula, es decir, existe correlación positiva entre las habilidades emocionales de los estudiantes venezolanos en el contexto investigado. Lo cual sugiere relación entre las inteligencias interpersonales e intrapersonal de Gardner (2001).

Tabla 4. Coeficientes de correlación de acuerdo con la prueba Rho de Spearman.

\begin{tabular}{|c|c|c|c|c|c|c|}
\hline $\begin{array}{l}\text { Habilidad } \\
\text { emocional }\end{array}$ & $\begin{array}{c}\text { Autocon } \\
\text {-ciencia } \\
1\end{array}$ & $\begin{array}{l}\text { Autocon- } \\
\text { ciencia } 2\end{array}$ & $\begin{array}{l}\text { Autocon- } \\
\text { trol }\end{array}$ & $\begin{array}{l}\text { Empa- } \\
\text { tía }\end{array}$ & $\begin{array}{l}\text { Moti- } \\
\text { vació } \\
n\end{array}$ & $\begin{array}{c}\text { Compete } \\
\text { n-cia } \\
\text { social }\end{array}$ \\
\hline \multirow{2}{*}{$\begin{array}{l}\text { Autoconcien- } \\
\text { cia } 1\end{array}$} & 1,000 & 0,488 & 0,347 & 0,496 & 0,458 & 0,466 \\
\hline & $* * * *$. & 0,000 & 0,000 & 0,000 & 0,000 & 0,000 \\
\hline \multirow{2}{*}{$\begin{array}{l}\text { Autoconcien- } \\
\quad \text { cia } 2\end{array}$} & 0,488 & 1,000 & $\underset{(}{0,484}$ & 0,531 & 0,552 & 0,509 \\
\hline & 0,000 & $* * * *$. & 0,000 & 0,000 & 0,000 & 0,000 \\
\hline \multirow{2}{*}{ Autocontrol } & 0,347 & 0,484 & 1,000 & 0,384 & 0,407 & 0,271 \\
\hline & 0,000 & 0,000 & $* * * *$. & 0,000 & 0,000 & 0,000 \\
\hline \multirow{2}{*}{ Empatía } & 0,496 & 0,531 & 0,384 & 1,000 & 0,471 & 0,518 \\
\hline & 0,000 & 0,000 & 0,000 & $* * * *$ & 0,000 & 0,000 \\
\hline Motivación & 0,458 & $0,552($ & 0,407 & 0,471 & 1,000 & 0,509 \\
\hline
\end{tabular}




\begin{tabular}{|c|c|c|c|c|c|c|c|} 
& & 0,000 & 0,000 & 0,000 & 0,000 & $* * * *$. & 0,000 \\
\hline \multirow{2}{*}{$\begin{array}{c}\text { Competencia } \\
\text { Social }\end{array}$} & 0,466 & 0,509 & 0,271 & 0,518 & 0,509 & 1,000 \\
\cline { 2 - 7 } & 0,000 & 0,000 & 0,000 & 0,000 & 0,000 & $* * * *$. \\
\hline
\end{tabular}

En relación a la hipótesis c. «Existen diferencias significativas entre las habilidades emocionales de los estudiantes por contexto geográfico».

Los promedios de las habilidades emocionales de los estudiantes de Los Teques fueron mayores que los de Caracas. Para determinar si estas diferencias en los promedios de las habilidades emocionales eran significativas, se aplicó la prueba de contraste de medianas de Mann-Whitney para dos muestras independientes. Los resultados de esta prueba indicaron que, entre los estudiantes de Caracas y los estudiantes de Los Teques, solo existen diferencias significativas en la habilidades emocionales de autoconciencia $1(p=0,021)$, autoconciencia $2(p=0,03)$ y autocontrol $(p=0,008)$. No se observaron diferencias significativas en las habilidades emocionales de empatía $(p=0,052)$, motivación $(p=$ $0,781)$ y competencia social $(p=0,117)$.

Tabla 5. Comparación entre las habilidades emocionales de estudiantes de Los Teques y de Caracas

\begin{tabular}{|c|c|c|c|c|c|c|c|c|}
\hline & \multicolumn{4}{|c|}{ Estudiantes de Caracas } & \multicolumn{4}{c|}{ Estudiantes de Los Teques } \\
\hline $\begin{array}{c}\text { Habilidad } \\
\text { emocional }\end{array}$ & $\mathrm{N}$ & Mediana & Media & $\sigma$ & $\mathrm{N}$ & Mediana & Media & $\sigma$ \\
\hline $\begin{array}{c}\text { Autoconcienci } \\
\text { a 1 }\end{array}$ & 247 & 19 & 19,11 & 3,29 & 185 & 20 & 19,89 & 3,07 \\
\hline $\begin{array}{c}\text { Autoconcienci } \\
\text { a 2 }\end{array}$ & 247 & 19 & 18,52 & 3,49 & 185 & 19 & 19,35 & 3,04 \\
\hline $\begin{array}{c}\text { Autocontrol } \\
\text { Empatía }\end{array}$ & 247 & 18 & 17,75 & 3,60 & 185 & 19 & 18,73 & 3,13 \\
\hline Motivación & 247 & 20 & 20,04 & 3,45 & 185 & 20 & 20,14 & 2,99 \\
\hline $\begin{array}{c}\text { Competencia } \\
\text { Social }\end{array}$ & 247 & 18 & 17,99 & 3,74 & 185 & 19 & 18,67 & 3,08 \\
\hline
\end{tabular}

Tabla 6. Estadísticos de prueba para la comparación de medianas entre las habilidades emocionales de los estudiantes de Los Teques y de Caracas 


\begin{tabular}{|l|c|c|c|c|c|c|}
\hline Habilidad emocional & $\begin{array}{c}\text { Autocon- } \\
\text { ciencia 1 }\end{array}$ & $\begin{array}{c}\text { Autocon- } \\
\text { ciencia 2 }\end{array}$ & $\begin{array}{c}\text { Auto- } \\
\text { control }\end{array}$ & Empatía & $\begin{array}{c}\text { Motiva- } \\
\text { ción }\end{array}$ & $\begin{array}{c}\text { Competen- } \\
\text { cia Social }\end{array}$ \\
\hline Ude Mann-Whitney & 19923,0 & 20102,5 & 19479,5 & 20393,0 & 22522,5 & 20872,0 \\
W de Wilcoxon & 50304,0 & 50483,5 & 49860,5 & 50774,0 & 39913,5 & 51253,0 \\
$Z$ & $-2,311$ & $-2,171$ & $-2,656$ & $-1,943$ & $-0,278$ & $-1,568$ \\
$p$ & 0,021 & 0,030 & 0,008 & 0,052 & 0,781 & 0,117 \\
\hline
\end{tabular}

Los estudiantes de Los Teques poseen mayores niveles de autoconciencia y autocontrol que los estudiantes de Caracas. Por tanto, la hipótesis de la investigación solo se cumple para las habilidades emocionales de autoconciencia y autocontrol, debido a que se observan diferencias significativas.

Entre los estudiantes de Los Teques, la prueba de Wilcoxon nos permitió determinar que las habilidades emocionales menos desarrollas fueron competencia social y autocontrol, no encontrándose diferencias significativas entre estas dos habilidades $(W=$ $7391,5, z=0,32957, p=0,74173)$. La prueba anterior aplicada a los estudiantes de Caracas no encontró diferencias significativas entre las habilidades emocionales de autocontrol y competencia social $(W=13313, z=0,737, p=0,461)$, siendo estas dos habilidades emocionales las menos desarrolladas.

\section{En relación a la hipótesis $d$. «Existen diferencias significativas entre las habilidades emocionales de estudiantes por género».}

Para contestar la hipótesis d hemos tomado 193 estudiantes de educación media y formación técnica superior de género femenino y 239 estudiantes de educación media y formación técnica superior de género masculino. Los estadísticos son mostrados en la tabla 7.

Salvo en autocontrol, los valores medios de las habilidades emocionales de los estudiantes de género femenino fueron mayores que los valores medios de los estudiantes de género masculino. Para determinar si estas diferencias eran significativas, se aplicó la prueba de Mann-Whitneypara dos muestras independientes. Los resultados de esta prueba indicaron que solo se observan diferencias significativas entre las habilidades emocionales de autoconciencia 1 ( $p=0,010)$, motivación $(p=0,018)$ y empatía $(p=0,04)$, es decir, los estudiantes de género femenino poseen mayores promedios en las habilidades emocionales de autoconciencia del primer nivel, motivación y empatía que los estudiantes de género 
masculino. No se encontraron diferencias significativas entre las habilidades emocionales de autoconciencia $2(p=0,065)$, autocontrol $(p=0,787)$ y competencia social $(p=0,825)$. Por tanto, la hipótesis de la investigación solo se cumple para las habilidades emocionales de autoconciencia 1, motivación y empatía, debido a que se observan diferencias significativas entre estas variables.

Tabla 7. Comparación de las habilidades emocionales de hombres y mujeres

\begin{tabular}{|c|c|c|c|c|c|c|c|c|}
\hline Género $\rightarrow$ & \multicolumn{4}{|c|}{ Hombres } & \multicolumn{4}{c|}{ Mujeres } \\
\hline $\begin{array}{c}\text { Habilidad } \\
\text { emocional }\end{array}$ & $\mathrm{N}$ & Mediana & Media & $\sigma$ & $\mathrm{N}$ & Mediana & Media & $\sigma$ \\
\hline $\begin{array}{c}\text { Autoconciencia } \\
1\end{array}$ & 239 & 19 & 18,78 & 3,76 & 19,3 & 20 & 19,75 & 3,00 \\
\hline $\begin{array}{c}\text { Autoconciencia } \\
2\end{array}$ & 239 & 19 & 18,47 & 3,38 & 193 & 19 & 19,08 & 3,13 \\
\hline Autocontrol & 239 & 18 & 18,18 & 3,45 & 193 & 18 & 18,04 & 3,49 \\
\hline Empatía & 239 & 18 & 18,45 & 3,41 & 193 & 20 & 19,15 & 3,30 \\
\hline Motivación & 239 & 20 & 19,73 & 3,31 & 193 & 21 & 20,63 & 3,00 \\
\hline $\begin{array}{c}\text { Competencia } \\
\text { Social }\end{array}$ & 239 & 18 & 18,09 & 3,54 & 193 & 18 & 18,22 & 3,40 \\
\hline
\end{tabular}

Tabla 8. Estadísticos de pruebapara la comparación de las habilidades emocionales de hombres y mujeres

\begin{tabular}{|l|c|c|c|c|c|c|}
\hline \multicolumn{1}{|c|}{ Estadísticos } & $\begin{array}{c}\text { Autocon- } \\
\text { ciencia 1 }\end{array}$ & $\begin{array}{l}\text { Autocon- } \\
\text { ciencia 2 }\end{array}$ & $\begin{array}{l}\text { Autocon- } \\
\text { trol }\end{array}$ & $\begin{array}{l}\text { Empa- } \\
\text { tía }\end{array}$ & $\begin{array}{l}\text { Motiva- } \\
\text { ción }\end{array}$ & $\begin{array}{l}\text { Competencia } \\
\text { Social }\end{array}$ \\
\hline$U$ de Mann-Whitney & 19759,0 & 20692,0 & 22716,0 & 20034,0 & 19416,5 & 22780,0 \\
\hline$W$ de Wilcoxon & 48439,0 & 49372,0 & 41437,0 & 48714,0 & 48096,5 & 51460,0 \\
\hline$Z$ & $-2,573$ & $-1,848$ &,- 270 & $-2,359$ & $-2,842$ & $-0,221$ \\
\hline $\begin{array}{l}p \\
\text { (bilateral) }\end{array}$ & 0,010 & 0,065 & 0,787 & 0,018 & 0,004 & 0,825 \\
\hline
\end{tabular}

Entre las estudiantes de género femenino, las habilidades emocionales menos desarrolladas fueron el autocontrol y la competencia social. Mediante la prueba de Wilcoxon no se observaron diferencias significativas entre estas dos habilidades emocionales $(W=7979,5, z=0,15129, p=0,8798)$.. Tampoco existen diferencias significativas entre autoconciencia 2 y empatía $(W=6594, z=0,1248, p=0,9006)$

Entre los estudiantes de género masculino, la prueba de Wilcoxon nos permitió determinar que las dos habilidades emocionales menos desarrolladas fueron también autocontrol y competencia social. Estas dos habilidades de los estudiantes de género 
masculino, solo se diferencian significativamente de las habilidades emocionales de autoconciencia del primer nivel y motivación (autocontrol-autoconciencia 1: $W=14255, z$ = 2,497, $p=0,013$; autocontrol-motivación: $W=5038,50, z=6,813, \quad p=0,000$; competencia social-autoconciencia $1: W=13128,50, z=2,894, p=0,004$; competencia social-motivación: $W=17679,50, z=7,180, p=0,000)$

En relación a la hipótesis e. «Los estudiantes de género femenino son emocionalmente más inteligentes que los de género masculino».

Para contestar la hipótesis e hemos utilizado los datos dados en las tablas 9 y 10 Las puntuaciones dadas en la columna de 75 percentiles de la tabla 9 fueron tomadas como el baremo para determinar la inteligencia emocional. Por ejemplo, un alumno para ser emocionalmente inteligente en la habilidad emocional de autocontrol, debe tener en el cuestionario de emociones una puntuación igual o mayor que 21. Para ser emocionalmente inteligente en la habilidad emocional de competencia social, debe obtener en el cuestionario de emociones una puntuación igual o superior a 20. De manera similar se interpretan los otros datos.

Tabla 9. Baremos para determinar la inteligencia emocional

\begin{tabular}{|l|c|c|c|c|}
\hline \multirow{2}{*}{ Habilidad emocional } & $\mathrm{N}$ & $\leq 25$ percentiles & 50 percentiles & $\geq 75$ percentiles \\
\cline { 3 - 5 } & & $\begin{array}{c}\text { Puntuación } \\
\text { Muy baja a baja }\end{array}$ & $\begin{array}{c}\text { Puntuación } \\
\text { Moderada }\end{array}$ & $\begin{array}{c}\text { Puntuación } \\
\text { Alta a muy alta }\end{array}$ \\
\hline Autoconciencia 1 & 432 & $0-17$ & $18-21$ & $22-25$ \\
\hline Autoconciencia 2 & 432 & $0-17$ & $18-21$ & $22-25$ \\
\hline Autocontrol & 432 & $0-16$ & $17-20$ & $21-25$ \\
\hline Empatía & 432 & $0-17$ & $18-20$ & $22-25$ \\
\hline Motivación & 432 & $0-18$ & $17-21$ & $20-25$ \\
\hline Competencia Social & 432 & $0-16$ & & 19 \\
\hline
\end{tabular}

Mediante un análisis de frecuencias por habilidad emocional se determinaron los porcentajes dados en la tabla 10. Mostramos, como un ejemplo, la interpretación de los 
datos de la tabla 10 para autoconciencia del primer nivel. En autoconciencia del primer nivel hubieron 58 estudiantes de género femenino emocionalmente inteligentes, que representa el 13,43\% y 54 estudiantes de género masculino emocionalmente inteligentes, que representa el $12,5 \%$. De manera similar se interpretan los otros datos.

Tabla 10. Número y porcentaje por género de estudiantes con puntuación $\geq 75$ percentile

\begin{tabular}{|c|c|c|c|c|c|c|}
\hline Género & $\begin{array}{c}\text { Autocon- } \\
\text { ciencia 1 }\end{array}$ & $\begin{array}{c}\text { Autocon- } \\
\text { ciencia 2 }\end{array}$ & $\begin{array}{c}\text { Auto- } \\
\text { control }\end{array}$ & $\begin{array}{c}\text { Empa- } \\
\text { tía }\end{array}$ & $\begin{array}{c}\text { Motiva- } \\
\text { ción }\end{array}$ & $\begin{array}{c}\text { Competen- } \\
\text { cia Social }\end{array}$ \\
\hline Femenino & 58 & 74 & 48 & 71 & 80 & 75 \\
\hline Masculino & 54 & 72 & 67 & 74 & 79 & 73 \\
\hline $\begin{array}{c}\% \\
\text { Femenino }\end{array}$ & 13,43 & 17,13 & 11,11 & 16,44 & 18,52 & 17,36 \\
\hline $\begin{array}{c}\% \\
\text { Masculino }\end{array}$ & 12,5 & 16,7 & 15,51 & 17,13 & 18,29 & 16,90 \\
\hline$\%$ Total & 25,93 & 33,83 & 26,62 & 33,57 & 36,81 & 34,26 \\
\hline
\end{tabular}

Salvo en autocontrol y empatía, los estudiantes de género femenino tienden a ser emocionalmente más inteligentes que los estudiantes de género masculino en cuatro de las seis habilidades emocionales. Por tanto, la hipótesis de la investigación se cumple para las habilidades emocionales de autoconciencia 1 y 2, motivación y competencia social.

\section{En relación a la hipótesis $f$. «Existe correlación lineal significativa entre las habilidades emocionales de los estudiantes y sus edades».}

Para contestar a la hipótesis f se determinaron los valores medios de las habilidades emocionales de los estudiantes, agrupados por edades de 15, 16, 17, 18 y mayores de 20 años. Se determinaron los coeficientes de correlación de Pearson de las líneas de tendencia, obtenidas al considerar la edad como variable independiente y el promedio de la habilidad emocional como variables dependiente. Los valores obtenidos para los coeficientes de correlación de las líneas de tendencia fueron los siguientes: autoconciencia $1, r=0,71(p=$ 0,16); autoconciencia 2, $r=0,65$ ( $p=0,21)$; autocontrol, $r=0,87$ ( $p=0,04)$; empatía, $r=$ $0,55(p=0,32)$; motivación, $r=0,58$ ( $p=0,28)$ y competencia social, $r=0,78(p=0,09)$. Como podemos apreciar, existe correlación lineal positiva entre las habilidades 
emocionales y la edad, pero solo la habilidad emocional de autocontrol posee relación lineal significativa. Por tanto, la hipótesis planteada solo se cumple para autocontrol.

\section{CONCLUSIONES}

En el contexto educativo investigado, las dos habilidades emocionales de menor promedio fueron autocontrol y competencia social, no encontrándose diferencias significativas entre los promedios de estas dos habilidades emocionales. Las diversas entrevistas e intercambios orales de impresión, realizadas con personal de aula y administrativa de amplia experiencia docente y las observaciones que hemos realizado diariamente en los salones de clase, son consistentes con los resultados reflejados en el cuestionario de emociones, es decir, nuestros alumnos poseen dificultades en el control de sus acciones y en el comportamiento social. También hemos notado que estudiantes con problemas disciplinarios y académicos tuvieron dificultades para contestar completamente el cuestionario de emociones.

Los estudiantes de los Teques tuvieron mayores promedios en las habilidades emocionales que los estudiantes de Caracas. El contraste de medias indicó que los estudiantes de Los Teques y los estudiantes de Caracas, se diferencian significativamente en las habilidades emocionales de autoconciencia y autocontrol. Este resultado sugiere que estudiantes venezolanos de diferentes zonas geográficas pueden poseer diferencias significativas en sus habilidades emocionales. En caso de desplazamiento forzoso, sugerimos que los padres preparen a sus hijos para adaptase a variaciones bruscas en el contexto geográfico y los efectos emocionales que se pueden producir.

Salvo en autocontrol, los estudiantes de género femenino tuvieron mayores promedios en las habilidades emocionales que los estudiantes de género masculino. El contraste de medias indicó diferencias significativas en las habilidades emocionales de autoconciencia 1, motivación y empatía, es decir, los estudiantes de género femenino poseen significativamente mayores promedios en estas tres habilidades emocionales que los estudiantes de género masculino. En el contexto educativo investigado y salvo en autocontrol y empatía, los estudiantes de género femenino tienden a ser emocionalmente más inteligentes que los estudiantes de género masculino. 
Hemos encontrado correlación lineal positiva entre los promedios de las habilidades emocionales y la edad de los estudiantes, pero solo la habilidad emocional de autocontrol presentó correlación lineal significativa. Es decir, nuestros estudiantes mejoran el autocontrol al pasar de la escuela secundaria a la universidad. Por tanto, debemos evitar que nuestros estudiantes abandonen sus estudios y garantizar un proceso educativo, continuo y permanente que mejore el bienestar personal y social de los mismos.

La relación observada entre las habilidades emocionales sugiere la existencia de relación positiva entre la inteligencia intrapersonal (autoconciencia, motivación y autocontrol) y la inteligencia interpersonal (empatía y competencia social).

La habilidad emocional con mayor puntuación fue motivación. No hemos podido encontrar relación significativa entre el promedio de notas de los alumnos y la habilidad emocional de motivación. Sobre la base de las observaciones realizadas en los salones de clase durante un período de tres años, sugerimos que deben mejorarse algunos factores de motivación intrínseca, tales como aumentar en los alumnos el deseo de mejorar en los aspectos que realmente importan, curiosidad, la necesidad de aprender, orden y la necesidad de tener un ambiente organizado y estable. Con referencia a lo anterior, la totalidad de los docentes consultados considera esencial actuar en el sentido de mejorar en nuestros alumnos las habilidades emocionales de autocontrol, competencia social y motivación intrínseca con miras a solventar problemas de disciplina y otros.

Por último, la información obtenida en esta investigación debe ser tomada como orientadora y nos servirá de reflexión para elaborar las estrategias apropiadas que permitan reforzar las habilidades emocionales de nuestros estudiantes. En este sentido, hemos venido estimulando la realización de proyectos de investigación, que envuelven el aprendizaje colaborativo, para potenciar la adquisición de habilidades emocionales interpersonales e intrapersonales. En la experiencia obtenida durante los dos últimos cursos académicos, se ha observado mejorías sustanciales en el rendimiento académico, colaboración entre los alumnos y entre alumnos-profesor. A través de la asignatura educación para la salud, impartida a estudiantes de $8^{\circ}$ grado, hemos suministrado conceptos básicos sobre habilidades emocionales. Los alumnos participantes han mostrado interés y entendimiento de los conceptos envueltos, pero es necesario un mayor número de clases, prácticas, generar 
las estrategias más adecuadas que se deben seguir y comprobar la efectividad real de las mismas.

\section{REFERENCIAS}

Alcázar, J. (2001). La inteligencia emocional. España: Editorial Limusa.

Alonso, C.M y Gallego, D. J. (2004, julio). Los Estilos de Aprendizaje: Una propuesta pedagógica. Ponencia presentada en el I Congreso Internacional de Estilos de Aprendizaje realizado en la UNED, Madrid, España.

Bisquerra, R. (2003). Educación emocional y competencias básicas para la vida. Revista de Investigación Educativa,21(1), 7-43. Disponible en: http://revistas.um.es/rie/article/view/99071/94661. [Consulta: 2016, Abril 9]

Burns, N. y Grove, S. (2005). The Practice of Nursing Research: Conduct, Critique, and Utilization (5th Ed.). St. Louis: Elsevier Saunders

Cabello, R., Ruiz-Aranda, D. y $\quad$ Fernández-Berrocal, P. (2010).Docentesemocionalmenteinteligentes.REIFOP, $\quad 13$ (1).Disponibleenhttp://www.aufop.com/aufop/uploaded_files/articulos/1268615555.pd f[Consulta: 2016, Julio 7]

Caruso, D. y Solovey, P. (2005). El directivo emocionalmente inteligente. La inteligencia emocional en la empresa. Málaga. Algaba.

Castejón, J.L., Cantero, P. y Pérez, N. (2008). Diferencias en el perfil de competencias socio-emocionales en estudiantes universitarios de diferentes ámbitos científicos. Electronic, Journal of Research in EducationalPsychology 6(2), 0-0. Disponible en: http://www.investigacion-psicopedagogica.org/revista/new/ContadorArticulo.php?267. [Consulta: 2016, Julio 8]

Elizondo, L. (2011). Competencias que debe tener un Director Académico Universitario para la Educación Superior Basada en Competencias. Revista de Investigación Educativa, 29(1), 205-218. Disponible en: http://revistas.um.es/rie/article/view/110791[Consulta: 2016, Agosto 7]

El Universal (2013). Aumentan muertes violentas entre jóvenes de 0 a 17 años. Disponible en: http:www.eluniversal.com/sucesos/130530//aumentan-muertes-violentas-entrejovenes-de-0-a-17-anos[Consulta: 2013, Agosto 3]

Fernández-Berrocal, P., Extremera, N. y Palomera, R. (2008). Emotional Intelligence as a crucial mental ability on educational context.En Valle, A. y Nuñez, J. C. (eds.), Handbook of Instructional Resources and their applications in the classroom. New York: Nova, 67-88.

Fernández-Berrocal, P. y Extremera, N. (2009). La Inteligencia Emocional y el estudio de la felicidad. Revista Interuniversitaria de Formación del Profesorado, 66(23,3),85-108. Disponible en: http://www.uned.es/ca- 
pamplona/IE\%20y\%20felicidad\%20Optimismo\%20monografico\%202009.pdf .[Consulta: 2016, Noviembre 10]

Gardner, H. (2001). Estructura de la Mente: La Teoría de Las Inteligencias Múltiples. Bogotá: Fondo de Cultura Económica LTDA.

Gil-Olarte, P., Palomera, R. y Brackett, M. A. (2006). Relating emotional intelligence to social competence and academic achievement in high school students.Psicothema, $18,118-123$

Gilar, R., Miñano, P. y Castejón, J.L. (2008).Inteligencia Emocional y Empatía: su influencia en la Competencia Social en Educación Secundaria obligatoria. SUMMA Psicológica UST, 5(1), 21-32.

Goleman, D. (1995). Emotional intelligence.Nueva York: Bantam Books.

Goleman, D. (1998). Working with emotional intelligence. Nueva York: Bantam Books.

Gutiérrez, M. (2010, octubre). La Inteligencia Emocional y los Estilos de Aprendizaje. Ponencia presentada en el IV Congreso Mundial de Estilos de Aprendizaje realizado en el Colegio de Postgraduados, Texcoco, México.

Kimbrough, G. (2008). Learning Styles and Emotional Intelligence of the Adult Learner.Alabama Auburn University: http://etd.auburn.edu/etd/bitstream/handle/10415/1066/Johnson_Gia_27.pdf?sequence $=1$ [Consulta: 2016, Agosto 7]

Lozano, J., Alcaraz, S. y Colás, P. (2010). Experiencia educativa sobre la comprensión de emociones y creencias en alumnado con trastornos del espectro autista. Revista de Investigación Educativa, 28(1), 65-78. Disponible en: http://revistas.um.es/rie/article/view/97931[Consulta: 2014, Agosto 7]

Molero, D., Ortega, F. y Moreno, MaRosario (2010). Diferencias en la adquisición de competencias emocionales en función del género.REID, 3,165-172. Disponible en: http://www.ujaen.es/revista/reid/revista/n3/REID3art9.pdf .[Consulta: 2016, Agosto 7]

Neptune, J. (2008). Importance of EQ skills for On-line Professors.The online journal, 6(4), 14. Disponible en http://connection.ebscohost.com/c/articles/43153305/importance-eqskills-on-line-professors.[Consulta: 2016, Septiembre 9]

Palomera, R., Fernández-Berrocal, P. y Brackett, M. A. (2008). La inteligencia emocional como una competencia básica en la formación inicial de los docentes: algunas evidencias. Revista Electrónica de Investigación Psicoeducativa,15 (6), 437-454. Disponible en: http://www.investigacionpsicopedagogica.org/revista/new/ContadorArticulo.php?276. [Consulta: 2016, Octubre 15]

Pena, M. y Repetto, E. (2008). Estado de la investigación en España sobre inteligencia emocional en el ámbito educativo. Revista Electrónica de Investigación Psicoeducativa,15 (6), 400-420.Disponible en: http://www.investigacionpsicopedagogica.org/revista/new/ContadorArticulo.php?244 . [Consulta: 2016, Octubre $18]$ 
Pérez, J.C., Petrides, K.V. y Furnham, A. (2007). Measuring trait emotional intelligence.En: R. Schulze y R.D. Roberts (Eds.), International Handbook of Emotional Intelligence (pp. 181-201). Cambridge, MA: Hogrefe\& Huber. Disponible en:

http://portal.uned.es/pls/portal/docs/PAGE/UNED_MAIN/LAUNIVERSIDAD/UBIC ACIONES/03/DOCENTE/JUAN_CARLOS_PEREZ_GONZALEZ/PEREZGONZALEZ,\%20PETRIDES,\%20\&\%20FURNHAM,\%202007_BOOKCHAPTER_SPANISH.PDF[Consulta: 2016, Noviembre 12]

Pérez, M.M. (2005)..Inteligencia emocional en el rol mediador del docente de inglés instrumental en la Universidad del Zulia. Maracaibo: Universidad del Zulia. Disponible en: http://tesis.luz.edu.ve/tde_busca/arquivo.php?codArquivo=827[Consulta: 2016, Noviembre 13]

Riggio, R. E. y Reichard, R. J. (2008). The emotional and social intelligences of effective leadership An emotional and social skill approach. Journal of Managerial Psychology,23(2),169-185.

Ruiz, D. (2008). Inteligencia Emocional y consumo de drogas en adolescentes. Málaga: Universidad de Málaga.

Salazar, W. (2002). Principios del paradigma cuantitativo en la investigación educativa. Revista de Ciencias del Ejercicio y la Salud, 2(1), 61-71

Salovey, P. y Mayer, J. D. (1990). Emotional intelligence Imagination.Cognition and Personality, 9 (3), 185-211.

Sampieri, R., Collado, C. y Lucio, P. (2008). Metodología de la Investigación. México: Mc Graw Hill.

Segura, J.M. (2011). Un estudio comparativo de las habilidades emocionales y los estilos de aprendizaje de estudiantes venezolanos de bachillerato y formación técnica superior. Revista Estilos de Aprendizaje, 8(8), 85-111. Disponible en: http://www.uned.es/revistaestilosdeaprendizaje/numero_8/sumario_completo/lsr_8_oct ubre_2011.pdf[Consulta: 2016, Noviembre 13]

Serrano, B. (2006). Inteligencia emocional: factor determinante en el rendimiento escolar. España: UNED

Singh, P. y Manser, P.G. (2008). Relationship between the perceived emotional intelligence of school principals and the job satisfaction of educators in a collegial environment.Disponibleen:

http://www.tandfonline.com/doi/abs/10.1080/18146620802144834[Consulta: 2016, Noviembre 10]

Soriano, E. y Osorio, M. M. (2008). Competencias socioemocionales del alumnado "autóctono" e inmigrante de educación secundaria. Bordón,60(1), 129-148.

Soriano, E. y Franco, C. (2010). Mejora de la autoestima y de la competencia emocional en adolescentes inmigrantes sudamericanos residentes en España a través de un programa psicoeducativo de Mindfulness (conciencia plena). Revista de Investigación Educativa, 
28 (2), 297-312. Disponible en: http://revistas.um.es/rie/article/view/99351/121601 . [Consulta: 2016, Noviembre 12]

Trujillo, M.M. y Rivas, L.A. (2005). Orígenes, evolución y modelos de inteligencia emocional.INNOVAR, 15(25), 9-24. Disponible en:http://www.revistas.unal.edu.co/index.php/innovar/article/viewFile/29/36[Consulta: 2016, Noviembre 12]

Universidad Pedagógica Experimental Libertador (2006). Manual de trabajos de grado, de especialización, maestría y tesis doctorales. Caracas: FEDUPEL

Vandervoort, D. (2006). The Importance of Emotional Intelligence in Higher Education.Current Psychology: Developmental Learning - Personality Social Spring, 25(1), 4-7. Disponible en: http://www.springerlink.com/content/bt85083772332160/fulltext.pdf?page=1.

[Consulta: 2016, Noviembre 12] 